\title{
Aortitis as a manifestation of myelodysplastic syndrome
}

\author{
F F Lopez, P B Vaidyan, A E Mega, F J Schiffman
}

\begin{abstract}
Aortitis is the inflammation of the wall of the aorta and can occur from an infection or autoimmune disease. Myelodysplastic syndrome (MDS) is characterised by abnormal haematopoiesis and a dysfunctional immune system. Autoimmune manifestations have been described in MDS. Here a case of a patient with aortitis and MDS is presented and discussed. All possible aetiologies were ruled out. The patient's symptoms resolved after she received steroids.

(Postgrad Med f 2001;77:116-118)
\end{abstract}

Keywords: aortitis; myelodysplastic syndrome; autoimmune

The Miriam Hospital, Brown University School of Medicine, Providence, Rhode Island, USA:

Department of Hematology/Oncology

F F Lopez

A E Mega

F J Schiffman

Department of

Medicine

P B Vaidyan

Correspondence to: Dr Anthony E Mega, The Miriam Hospital/Brown University, 164 Summit Avenue, Providence, RI 02906, USA

Amega@Lifespan.org

Submitted 8 March 2000 Accepted 5 June 2000

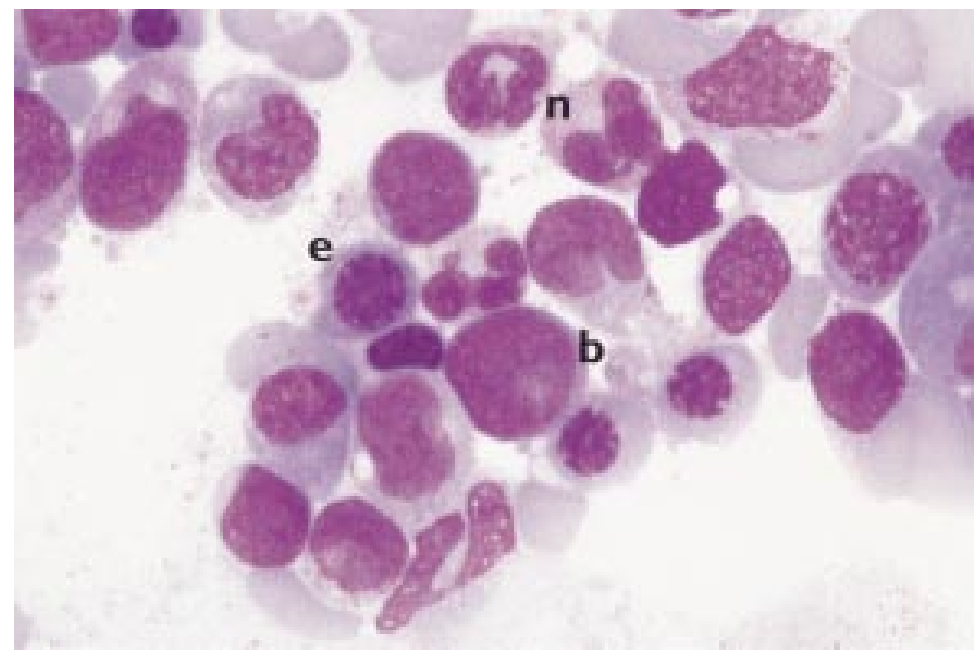

Figure 1 Bone marrow aspirate showing dysplastic neutrophils (n), megaloblastic erythroid (e) precursors, and blast (b) cells.
She had no significant medical and social history. Physical examination was as follows. She was well nourished and in no distress. Blood pressure was $120 / 70 \mathrm{~mm} \mathrm{Hg}$ in both upper extremities, heart rate 92 beats/min, respirations 20 breaths/min, and temperature of $102^{\circ} \mathrm{F}$. She had no lymph node enlargement or distended jugular veins. Chest and lung examination were significant for decreased breath sounds over the left lower lung fields with overlying costal and sternal tenderness. Spleen and liver were not palpable. Peripheral pulses were full and equal in all extremities.

Several diagnostic tests were done to investigate the cause of the chest pain. Myocardial infarction was considered and ruled out with negative results on three blood tests for creatine phosphokinase and MB isoenzymes. Furthermore, she had a 12-lead electrocardiogram with sinus tachycardia and low voltage complexes and a normal echocardiogram. Pulmonary embolism was entertained. However, arterial blood gas on room air was normal and ventilation/perfusion scan revealed a low probability. Chest radiography revealed minimal infiltrate in the left lower lung field and blunting of the left costophrenic angle. Hence, her chest pain was most likely due to an infectious pneumonia. This working diagnosis was strengthened by the fact that she was pancytopenic with a white blood cell count of $3.2 \times$ $10^{9} / 1$ and normal differential, haemoglobin of $97 \mathrm{~g} / \mathrm{l}$, mean corpuscular volume of $104 \mathrm{fl}$, platelet count of $660 \times 10^{9} / 1$, and reticulocyte count of $1 \%$. Peripheral smear was notable for a leukoerythroblastic picture. Bone marrow biopsy and aspiration showed dyserythropoietic erythroid and myeloid cells with more than $20 \%$ blasts (fig 1). These findings were consistent with refractory anaemia with excess blasts in transformation (RAEB-t). Cytogenetic analysis was normal.

The patient was treated with intravenous antibiotics. However, her symptoms worsened. Sputum and blood cultures remained negative. Repeat chest radiography showed increasing left pleural effusion. Thoracentesis was performed and pleural fluid analysis was exudative. No malignant cells were present. Cultures for bacteria, acid-fast bacillus, and fungus were negative. Since the pleural fluid analysis was non-diagnostic, the patient had computed tomography followed by a magnetic resonance imaging (MRI) of the chest. Both revealed aortic wall thickening with dilatation and paraaortic fluid collection involving the arch and descending thoracic aorta consistent with aortitis (fig 2). These radiographic findings plus a raised erythrocyte sedimentation rate of 120 $\mathrm{mm} /$ hour concur with an inflammatory process 


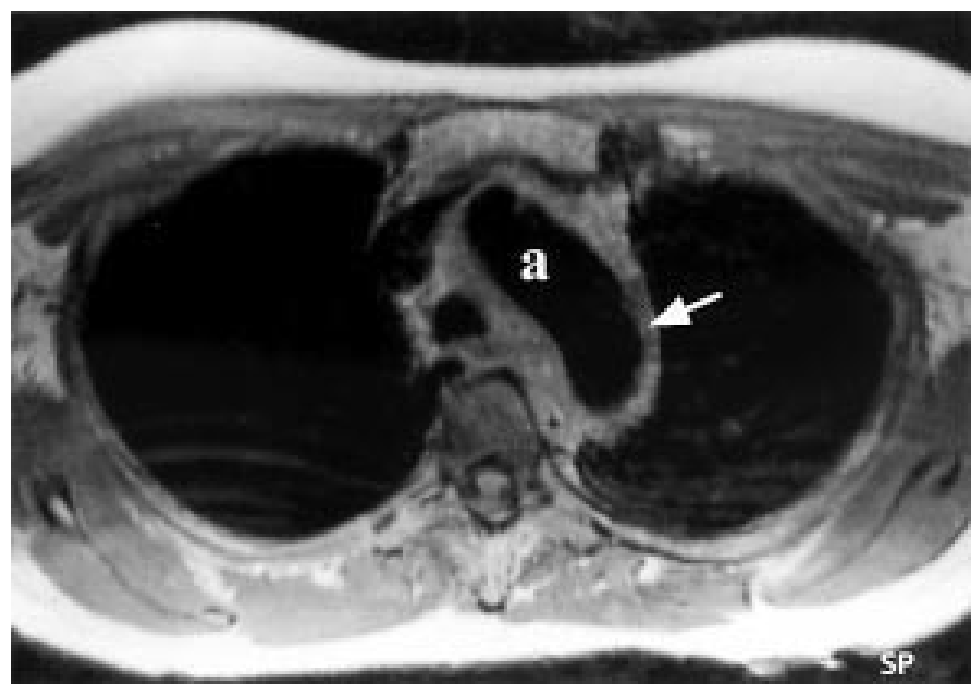

Figure 2 MRI axial T1 weighted image of the chest shows arrow pointing at thickened aorta (a), representing aortitis.

contributing to a reactive and exudative pleural effusion. Serology was negative for rapid plasma reagin, antinuclear antibody, rheumatoid factor, and antineutrophil cytoplasmic antibodies. The patient was started on methylprednisone $60 \mathrm{mg}$ intravenous daily then prednisone $60 \mathrm{mg}$ oral daily. Her condition dramatically improved and pleural effusion resolved within a few days. Several months later she underwent a matched unrelated donor bone marrow transplantation. The transplant was without any complications. However, she developed an intra-abdominal infection several months later and expired.

\section{Discussion}

The aetiology of aortitis can be classified as infectious or immune mediated. Infectious agents such as Treponema pallidum, Salmonella $\mathrm{sp}$, Staphylococcus aureus, candida, and Mycobacterium tuberculosis have been implicated. Microorganisms gain a foothold in the aortic wall through seeding, direct invasion, septic emboli, contiguous spread, and trauma. ${ }^{1}$ Rheumatological diseases such as Takayasu's arteritis, giant cell arteritis, and HLA-B27 associated spondylarthropathies are possible causes of aortitis. The diagnosis of aortitis is established by performing a radiographic procedure such as computed tomography, MRI, or angiography in a clinical setting suspicious of these diseases.

MDS is a heterogenous group of disorders characterised by dyserythropoiesis and peripheral cytopenia. The patient may be completely asymptomatic, exhibit symptoms of anaemia, or present with an infection. The full blood count typically shows a macrocytic anaemia, bilobed neutrophils, or pancytopenia. The diagnosis is confirmed by performing a bone marrow aspirate. The French-American-British system distinguishes five entities. These are refractory anaemia, refractory anaemia with ringed sideroblasts (RARS), refractory anaemia with excess blasts (RAEB) if the marrow shows 5\%-20\% blasts, RAEB in transformation (RAEB-t) if the marrow shows $20 \%-30 \%$ blasts, and chronic myelomonocytic leukaemia if the

\section{Learning points}

- Acute systemic vasculitis occurs in all subgroups of MDS.

- Presence of an autoimmune disease is associated with very rapid clinical deterioration and short survival regardless of subgroup.

- Immunological abnormalities include hypogammmaglobulinaemia or hypergammmaglobulinaemia, functionally immature B cells, reduced CD4 positive cells and impaired $T$ cell function, reduced and functionally immature natural killer cells, and impaired monocyte function.

- Systemic vasculitis associated with MDS respond to immunosuppressive therapy.

peripheral blood count shows monocyte count greater than $1000 / \mu$ l. The elderly patients with refractory anaemia and RARS can survive for several years with supportive care such as blood transfusions, granulocyte colony stimulating factor, or erythropoeitin. On the other hand, the patients who present with RAEB-t will eventually develop acute myelogenous leukaemia within 30 months. Hence, allogeneic or matched unrelated donor bone marrow transplantation is offered to these patients, especially if they are younger.

Published case reports have documented an association of immunological manifestations and $\mathrm{MDS}^{2}{ }^{3}$ To name a few, leukocytoclastic vasculitis, polyarthritis, pleuropericarditis, Raynaud's syndrome, and relapsing polychondritis have been described. Recently, Enright and Miller identified $13.6 \%$ of patients with MDS who also had clinical autoimmune disease. ${ }^{4}$ They classified these clinical autoimmune manifestations into three broad categories, namely: acute systemic vasculitis, chronic/isolated autoimmune and classic connective tissue disorders. Acute systemic vasculitis occurs in all subgroups of MDS. The diagnosis of MDS may precede or occur with the autoimmune manifestations. The presence of an autoimmune disease is associated with very rapid clinical deterioration and a median survival of only six months. Causes of death included respiratory failure secondary to worsening pulmonary infiltrates, intracranial haemorrhage associated with a flare of the systemic vasculitic syndrome, gastrointestinal haemorrhage, and sepsis. The underlying immunological abnormalities in MDS include hypergammaglobulinaemia or hypogammaglobulinaemia, functionally immature B cells, reduced CD4 positive cells ${ }^{4}$ and impaired $T$ cell function, reduced and functionally immature natural killer cells, and impaired monocyte function. ${ }^{56}$ Consequently, phagocytic function is impaired. Immune complexes that are normally disposed of are deposited in small blood vessels allowing the local activation of inflammatory mediators. ${ }^{5}$ Lymphocytes or monocytes release lymphokines that perturb the endothelial cell lining of blood vessels in the skin, synovium, peripheral nerves, or other organs. This 
immunological imbalance may predispose to the development of vasculitis. Prednisone 1-2 mg/ $\mathrm{kg}$ /day, methylprednisolone 1-2 $\mathrm{g}$ bolus doses, or other immunosuppressive agents including cyclophosphamide and azathiroprine is effective in resolving these manifestations. However, these medications are not effective in the treatment of MDS.

Our patient had a pleuritic chest pain, reactive inflammatory pleural effusion and a raised erythrocyte sedimentation rate. These manifestations of an acute vasculitic syndrome, in this case aortitis, occurred simultaneously with the diagnosis of MDS subgroup RAEB-t. Her symptoms dramatically improved after administration of steroids. The resolution of her symptoms further strengthens the observation of an acute vasculitic syndrome which is due to an abnormal immune system in MDS.
Aortitis should be included as part of the expanding list of autoimmune manifestations in MDS. Conversely, MDS should be considered as a possible aetiology of aortitis in the absence of any underlying infectious or rheumatological disease.

1 Lindsay J Jr. Diagnosis and treatment of disease of the aorta. Curr Probl Cardiol 1997;22:485-542.

2 Castro M, Conn D, Su WP, et al. Rheumatic manifestations Castro M, Conn D, Su WP, et al. Rheumatic manifestations
in myelodysplastic syndromes. $\mathcal{F}$ Rheumatol 1991;18:721-7. 3 George S, Newman E. Seronegative inflammatory arthritis in the myelodysplastic syndromes. Semin Arthritis Rheum 1992;21:345-54.

4 Enright H, Miller W. Autoimmune phenomena in patients with myelodysplastic syndromes. Leuk Lymphoma 1997;24: with m.

5 Okamoto T, Okada M, Mori A, et al. Correlation between immunological abnormalities and prognosis in myelodysplastic syndrome patients. Int $\mathcal{F}$ Hematol 1997;66:345-51.

6 Hamblin T. Immunological abnormalities in myelodysplastic syndrome. Semin Hematol 1996;33:150-62. 